International Journal of Pure and Applied Mathematics

Volume 84 No. 4 2013, 331-343

ISSN: 1311-8080 (printed version); ISSN: 1314-3395 (on-line version)

url: http://www.ijpam.eu

doi: http://dx.doi.org/10.12732/ijpam.v84i4.3

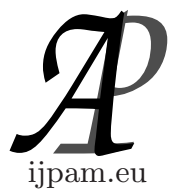

\title{
MODIFIED OPTIMAL FAMILIES OF FOURTH-ORDER JARRATT'S METHOD
}

\author{
Ramandeep Behl ${ }^{1}$, V. Kanwar ${ }^{2}$, Kapil K. Sharma ${ }^{3}$ \\ ${ }^{1,3}$ Department of Mathematics \\ Panjab University \\ Chandigarh, 160 014, INDIA \\ ${ }^{2}$ University Institute of Engineering and Technology \\ Panjab University \\ Chandigarh, 160 014, INDIA
}

\begin{abstract}
In this paper, we present a two parameter modified families of Jarratt's method having quartic convergence for computing simple roots of nonlinear equations, permitting $f^{\prime}(x)=0$ in the vicinity of the required root. The present approach of deriving this optimal class of Jarratt's method is based on applying weight function approach. Jarratt's method and Zhou et al. family of methods [1] for simple roots can be seen as special cases of our proposed scheme. Each member of the class requires three functional evaluations per iteration. The performance of proposed multipoint methods is compared with its closest competitors namely, King's method, Ostrowski's method and Jarratt's method in a series of numerical experiments. All the methods considered here are found to be more effective and comparable to the similar robust methods available in the literature.
\end{abstract}

AMS Subject Classification: 65xx, 65Hxx

Key Words: nonlinear equations, simple roots, Newton's method, Jarratt's method, optimal order of convergence, efficiency index

Received: October 26, 2012

(C) 2013 Academic Publications, Ltd.

$\S$ Correspondence author url: www.acadpubl.eu 


\section{Introduction}

This paper addresses the problem of finding simple roots of nonlinear equations of the form

$$
f(x)=0,
$$

where $f: D \subset \mathbb{R} \rightarrow \mathbb{R}$ be a nonlinear continuous function on $D$. Analytical methods for solving such equations are almost non-existent and therefore, it is only possible to obtain approximate solutions by relying on numerical methods based on iterative procedures. There are several one-point and multipoint iterative methods available in the literature [1-11]. Newton's method for solving equation (1.1) is given by

$$
x_{n+1}=x_{n}-\frac{f\left(x_{n}\right)}{f^{\prime}\left(x_{n}\right)} .
$$

It is probably the best known and most widely used algorithm for solving such problems. It converges quadratically to a simple root and linearly to a multiple root. However, a major difficulty in the application of Newton's method is the selection of initial guess such that neither guess is far from zero nor the derivative is small in the vicinity of the required root, otherwise the method fails miserably. Finding a criterion for choosing initial guess is quite cumbersome and therefore, more effective globally convergent algorithms are still needed. In order to overcome this problem, Kumar et. al. [2] have proposed the following one-point iterative scheme given by

$$
x_{n+1}=x_{n}-\frac{f\left(x_{n}\right)}{f^{\prime}\left(x_{n}\right)-p f\left(x_{n}\right)} .
$$

This scheme is derived by implementing approximations through a straight line in the vicinity of required root. This family converges quadratically under the condition $f^{\prime}\left(x_{n}\right)-p f\left(x_{n}\right) \neq 0$, while $f^{\prime}\left(x_{n}\right)=0$ is permitted at some points. For $p=0$, we obtain Newton's method. The error equation of scheme (1.3) is given by

$$
e_{n+1}=\left(p-c_{2}\right) e_{n}^{2}+O\left(e_{n}^{3}\right) \text {, }
$$

where $e_{n}=x_{n}-r, c_{k}=\frac{1}{k !} \frac{f^{(k)}(r)}{f^{\prime}(r)}, k=2,3, \ldots$ and $x=r$ is the root of nonlinear equation (1.1). In order to obtain quadratic convergence, the entity in the denominator should be largest in magnitude. Further, it can be seen that this family of Newton's method gives very good approximation to the root when $|p|$ is small. This is because that, for small values of $p$, slope or angle of 
inclination of straight line with $x$-axis becomes smaller, i.e. as $p \rightarrow 0$, the straight line tends to $x$ - axis.

Multipoint iterative methods for solving nonlinear equations play a significant role in the field of iterative processes since they circumvent the drawbacks of one-point iterations, such as Newton's method. Such constructions occasionally possess a better order of convergence and efficiency index for finding the simple or multiple roots. In past and recent years, many multipoint iterative methods have been proposed for solving nonlinear equations that improve local convergence order of the classical Newton's method, see [1-8]. In 1969, Jarratt [4] considered the following multipoint iterative scheme for simple roots:

$$
\left\{\begin{aligned}
y_{n} & =x_{n}-\frac{2}{3} \frac{f\left(x_{n}\right)}{f^{\prime}\left(x_{n}\right)}, \\
x_{n+1} & =x_{n}-\frac{f\left(x_{n}\right)}{2 f^{\prime}\left(x_{n}\right)}\left[\frac{3 f^{\prime}\left(y_{n}\right)-2 f^{\prime}\left(x_{n}\right)}{3 f^{\prime}\left(y_{n}\right)+2 f^{\prime}\left(x_{n}\right)}\right] .
\end{aligned}\right.
$$

This method has optimal fourth-order convergence according to the KungTraub conjecture [5] and one of the most efficient fourth-order method known to date. However, this method is a variant of Newton's method and the iteration can be aborted due to overflow or leads to divergence, if the derivative of function at an iterative point is singular or almost singular, which restrict its applications in practical.

Therefore, construction of an optimal class of Jarratt's method having quartic convergence and converges to the required root even though the guess is far from zero or the derivative is small in the vicinity of the required root is an open and challenging problem in computational mathematics. With this aim, we intend to construct a globally convergent general class of Jarratt's method. The present approach of deriving this optimal class of Jarratt's method is based on applying weight function approach on Kumar et al.'s scheme [2]. All the proposed methods considered here are found to be effective and comparable to the classical Jarratt's method.

The contents of this paper unfold the material in what follows. Section 2 presents the development of the general class of Jarratt's method, which will converge in case the initial guess is far from zero or the derivative is small in the vicinity of the required root. In section 3, we describe some specific cases of our proposed general class. Section 4 includes a numerical comparison between proposed methods without memory and the existing robust Jarratt's method and finally, the concluding remarks of the paper have been drawn. 


\section{Construction of Novel Techniques without Memory}

In this section, we intend to develop a new optimal general class of Jarratt's method which will converge even though $f^{\prime}(x)=0$. For this purpose, we extend the idea of Kumar et al. [2] and consider the following two-step scheme as follows:

$$
\left\{\begin{array}{c}
y_{n}=x_{n}-a \frac{f\left(x_{n}\right)}{f^{\prime}\left(x_{n}\right)-p f\left(x_{n}\right)}, \\
x_{n+1}=x_{n}-\frac{f\left(x_{n}\right)}{f^{\prime}\left(x_{n}\right)-p f\left(x_{n}\right)} Q\left(\frac{f^{\prime}\left(y_{n}\right)+h f^{\prime}\left(x_{n}\right)}{t f^{\prime}\left(x_{n}\right)-p f\left(x_{n}\right)}\right),
\end{array}\right.
$$

where $a, p, t$ and $h$ are four free disposable parameters such that the order of convergence reaches at the optimal level four without using any more functional evaluations. Theorem 2.1 indicates that under what conditions on the disposable parameters in (2.1), the order of convergence will reach at the optimal level four.

Theorem 2.1. Let $f: I \subseteq \mathbb{R} \rightarrow \mathbb{R}$ has atleast fourth continuous derivatives defined on an open interval $I$, enclosing a simple zero of $f(x)$ (say $x=r \in I$ ). Assume that initial guess $x=x_{0}$ is sufficiently close to $r$ and $f^{\prime}\left(x_{n}\right)-p f\left(x_{n}\right) \neq 0$ in $I$. Then the family of iterative methods defined by (2.1) has fourth-order convergence when

$$
\left\{\begin{array}{l}
Q(\mu)=1, \\
Q^{\prime}(\mu)=-\frac{3}{8}, \\
Q^{\prime \prime}(\mu)=\frac{9}{16}, \\
t=\frac{1}{2} \\
a=\frac{2}{3} \\
h=-\frac{1}{3}
\end{array}\right.
$$

where $\mu=\frac{4}{3}$ and satisfies the following error equation

$$
e_{n+1}=\left(\left(c_{2}-p\right)\left(4 p^{2}+\left(5 c_{2}^{2}-c_{3}-9 p c_{2}\right)\right)+\frac{c_{4}}{9}\right) e_{n}^{4}+O\left(e_{n}^{5}\right),
$$

where $e_{n}=x_{n}-r$ and $c_{k}=\frac{1}{k !} \frac{f^{(k)}(r)}{f^{\prime}(r)}, k=2,3, \ldots$ 
Proof. Let $x=r$ be a simple zero of $f(x)$. Expanding $f\left(x_{n}\right)$ and $f^{\prime}\left(x_{n}\right)$ about $x=r$ by the Taylor's series expansion, we have

$$
f\left(x_{n}\right)=f^{\prime}(r)\left(e_{n}+c_{2} e_{n}^{2}+c_{3} e_{n}^{3}+c_{4} e_{n}^{4}\right)+O\left(e_{n}^{5}\right),
$$

and

$$
f^{\prime}\left(x_{n}\right)=f^{\prime}(r)\left(1+2 c_{2} e_{n}+3 c_{3} e_{n}^{2}+4 c_{4} e_{n}^{3}\right)+O\left(e_{n}^{4}\right),
$$

respectively.

From equations (2.4) and (2.5), we have

$$
\begin{aligned}
& \frac{f\left(x_{n}\right)}{f^{\prime}\left(x_{n}\right)-p f\left(x_{n}\right)}=e_{n}+\left(p-c_{2}\right) e_{n}^{2}+\left(p^{2}-2 p c_{2}+2 c_{2}^{2}-2 c_{3}\right) e_{n}^{3} \\
& \quad+\left(p^{3}+5 p c_{2}^{2}-4 c_{2}^{3}-4 c_{2}^{3}-4 p c_{3}+c_{2}\left(7 c_{3}-3 p^{2}\right)-3 c_{4}\right) e_{n}^{4}+O\left(e_{n}^{5}\right),
\end{aligned}
$$

and in the combination of Taylor series expansion of $f^{\prime}\left(x_{n}-a \frac{f\left(x_{n}\right)}{f^{\prime}\left(x_{n}\right)-p f\left(x_{n}\right)}\right)$ about $x=r$, we have

$$
\begin{aligned}
f^{\prime}\left(y_{n}\right) & =f^{\prime}\left(x_{n}-a \frac{f\left(x_{n}\right)}{f^{\prime}\left(x_{n}\right)-p f\left(x_{n}\right)}\right) \\
& =f^{\prime}(r)\left[1+2(1-a) c_{2} e_{n}+\left(2 a c_{2}^{2}-2 a p c_{2}+3(a-1)^{2} c_{3}\right) e_{n}^{2}\right. \\
& -2\left(2 a c_{2}^{3}-2 a p c_{2}^{2}+a c_{2}\left(p^{2}+(3 a-5) c_{3}\right)+(a-1)\left(2(a-1)^{2} c_{4}\right.\right. \\
& \left.\left.\left.-3 a p c_{3}\right)\right) e_{n}^{3}\right]+O\left(e_{n}^{4}\right) .
\end{aligned}
$$

Furthermore, we have

$$
\begin{aligned}
& \frac{f^{\prime}\left(y_{n}\right)+h f^{\prime}\left(x_{n}\right)}{t f^{\prime}\left(x_{n}\right)-p f\left(x_{n}\right)}=\left(\frac{1+h}{t}\right)+\left(\frac{(1+h) p-2 a t c_{2}}{t^{2}}\right) e_{n} \\
& +\left(\frac{(1+h) p^{2}-p t(1+h+2 a(1+t)) c_{2}+6 a t^{2} c_{2}^{2}+3 a(a-2) t^{2} c_{3}}{t^{3}}\right) e_{n}^{2} \\
& +\frac{1}{t^{4}}\left[p^{3}(1+h)+2 p t^{2}\left(1+h+4 a(1+t)+8 a t c_{2}\right) c_{2}^{2}-p t^{2}(2(1+h)\right. \\
& \left.+6 a(1+t)-3 a^{2}(1+2 t)\right) c_{3}-2 t c_{2}\left(p^{2}(1+h)+a\left(1+b t+t^{2}\right)\right) \\
& \left.+2 a(3 a-7) t^{2} c_{3}-4 a^{3} t^{3} c_{4}+12 a t^{3}(a-1) c_{4}\right] e_{n}^{3}+O\left(e_{n}^{4}\right) .
\end{aligned}
$$

Since it is clear from $(2.8)$ that $\left(\frac{f^{\prime}\left(y_{n}\right)+h f^{\prime}\left(x_{n}\right)}{t f^{\prime}\left(x_{n}\right)-p f\left(x_{n}\right)}\right)-\mu$ is of order $e_{n}$, where $\mu=$ $\left(\frac{1+h}{t}\right)$. Hence, we can consider the Taylor's expansion of the weight function $Q$ in the neighborhood of $\mu$. Therefore, we have

$$
\begin{aligned}
Q\left(\frac{f^{\prime}\left(y_{\mathrm{n}}\right)+h f^{\prime}\left(x_{\mathrm{n}}\right)}{t f^{\prime}\left(x_{\mathrm{n}}\right)-p f\left(x_{\mathrm{n}}\right)}\right)= & Q(\mu)+\left(\frac{f^{\prime}\left(y_{\mathrm{n}}\right)+h f^{\prime}\left(x_{\mathrm{n}}\right)}{t f^{\prime}\left(x_{\mathrm{n}}\right)-p f\left(x_{\mathrm{n}}\right)}\right) Q^{\prime}(\mu) \\
& +\frac{1}{2 !}\left(\frac{f^{\prime}\left(y_{\mathrm{n}}\right)+h f^{\prime}\left(x_{\mathrm{n}}\right)}{t f^{\prime}\left(x_{\mathrm{n}}\right)-p f\left(x_{\mathrm{n}}\right)}\right)^{2} Q^{\prime \prime}(\mu)+O\left(e_{\mathrm{n}}^{3}\right) .
\end{aligned}
$$


Using (2.6), (2.8)and (2.9) in the scheme (2.1), we have the following error equation

$$
\begin{aligned}
e_{n+1} & =e_{n}-\frac{f\left(x_{n}\right)}{f^{\prime}\left(x_{n}\right)-p f\left(x_{n}\right)} Q\left(\frac{f^{\prime}\left(y_{n}\right)+h f^{\prime}\left(x_{n}\right)}{t f^{\prime}\left(x_{n}\right)-p f\left(x_{n}\right)}\right) \\
& =(1-Q(\mu)) e_{n} \\
& +\left(\frac{t\left(2 a Q^{\prime}(\mu)+t Q(\mu)\right) c_{2}-p\left((1+h) Q^{\prime}(\mu)+t^{2} Q(\mu)\right)}{t^{2}}\right) e_{n}^{2} \\
& +\left(\frac{A_{1}+A_{2} c_{2}-A_{3} c_{3}}{2 t^{4}}\right) e_{n}^{3}+O\left(e_{n}^{4}\right)
\end{aligned}
$$

where

$$
\begin{aligned}
& A_{1}=-p^{2}\left(2(1+h) t^{2} Q^{\prime}(\mu)+2 t^{4} Q(\mu)+(1+h)\left(2 t Q^{\prime}(\mu)+(1+h) Q^{\prime \prime}(u)\right)\right), \\
& A_{2}=+4 p t\left(b t(1+h) Q^{\prime}(\mu)+t^{2} Q(\mu)\right)+a\left(2 t^{2} Q^{\prime}(\mu)+\left(t Q^{\prime}(\mu)+(1+h) Q^{\prime \prime}(\mu)\right)\right), \\
& A_{3}=4 t^{2}\left(t^{2} Q(\mu)+4 a t Q^{\prime}(\mu)+a^{2} Q^{\prime \prime}(\mu)\right) c_{2}^{2}+2 t^{3}\left(2 t Q(\mu)+3 a(2-a) Q^{\prime}(u)\right) .
\end{aligned}
$$

For obtaining an optimal general class of fourth-order iterative methods, the coefficients of $e_{n}, e_{n}^{2}$, and $e_{n}^{3}$ in the error equation (2.10) must be zero simultaneously. After simplifying the equation (2.10), we have the following equations involving of $Q(\mu), Q^{\prime}(\mu)$, and $Q^{\prime \prime}(\mu)$

$$
\left\{\begin{array}{l}
Q(\mu)=1, \\
2 a Q^{\prime}(\mu)=t Q(\mu), \\
A_{1}=0 \\
A_{2}=0 \\
A_{3}=0
\end{array}\right.
$$

respectively.

Solving the above equations for $Q(\mu), Q^{\prime}(\mu), Q^{\prime \prime}(\mu), t, a$, and $h$, we get

$$
\left\{\begin{array}{l}
Q(\mu)=1, \\
Q^{\prime}(\mu)=-\frac{3}{8}, \\
Q^{\prime \prime}(\mu)=\frac{9}{16}, \\
t=\frac{1}{2}, \\
a=\frac{2}{3}, \\
h=-\frac{1}{3},
\end{array}\right.
$$


where $\mu=\frac{4}{3}$.

Using the above conditions, the scheme (2.1) will satisfy the following error equation

$$
e_{n+1}=\left(\left(c_{2}-p\right)\left(4 p^{2}+\left(5 c_{2}^{2}-c_{3}-9 p c_{2}\right)\right)+\frac{c_{4}}{9}\right) e_{n}^{4}+O\left(e_{n}^{5}\right),
$$

where $p \in \mathbb{R}$ is a free disposable parameter.

This reveals that the general two-step class of Jarratt's method (2.1) reaches the optimal order of convergence four by using only three functional evaluations per full iteration. The beauty of our proposed optimal general class of Jarratt's method is that it will converge to the required root even $f^{\prime}(x)=0$ unlike Jarratt's method. This completes the proof of the Theorem 2.1.

\section{Some Special Cases}

Finally, by using specific values of $a, t$, and $h$, which are defined in Theorem 2.1, we get the following general class of Jarratt's type iterative methods given by

$$
\left\{\begin{array}{c}
y_{n}=x_{n}-\frac{2}{3} \frac{f\left(x_{n}\right)}{f^{\prime}\left(x_{n}\right)-p f\left(x_{n}\right)}, \\
x_{n+1}=x_{n}-\frac{f\left(x_{n}\right)}{f^{\prime}\left(x_{n}\right)-p f\left(x_{n}\right)} Q\left(\frac{6 f^{\prime}\left(y_{n}\right)-2 f^{\prime}\left(x_{n}\right)}{3 f^{\prime}\left(x_{n}\right)-6 p f\left(x_{n}\right)}\right),
\end{array}\right.
$$

where $Q\left(\frac{6 f^{\prime}\left(y_{n}\right)-2 f^{\prime}\left(x_{n}\right)}{3 f^{\prime}\left(x_{n}\right)-6 p f\left(x_{n}\right)}\right)$ is a weight function which satisfies the conditions defined in Theorem 2.1. Again in (3.1), $p$ is chosen as a positive or negative sign so as to make the denominator largest in magnitude. Now, we shall consider some particular cases of the proposed scheme (3.1) depending upon the weight function $Q(x)$ and $p$ as follows:

Case 1. Let us consider the following weight function

$$
Q(x)=\frac{1}{2}+\frac{2}{3 x} \text {. }
$$

It can be easily seen that the above mentioned weight function $Q(x)$ satisfies all the conditions of Theorem 2.1. Therefore, we obtain a new optimal general class of fourth-order Jarratt's method given by

$$
\left\{\begin{array}{c}
y_{n}=x_{n}-\frac{2}{3} \frac{f\left(x_{n}\right)}{f^{\prime}\left(x_{n}\right)-p f\left(x_{n}\right)}, \\
x_{n+1}=x_{n}-\frac{f\left(x_{n}\right)}{f^{\prime}\left(x_{n}\right)-p f\left(x_{n}\right)}\left[\frac{1}{2}+\frac{2}{3}\left(\frac{3 f^{\prime}\left(x_{n}\right)-6 p f\left(x_{n}\right)}{6 f^{\prime}\left(y_{n}\right)-2 f^{\prime}\left(x_{n}\right)}\right)\right] .
\end{array}\right.
$$


This is a new general class of fourth-order optimal methods and one can easily get many new methods by choosing the different values of the disposable parameter $p$.

For example special case of optimal family (3.3): (i) For $p=0$, family (3.3) reads as

$$
\left\{\begin{aligned}
y_{n} & =x_{n}-\frac{2}{3} \frac{f\left(x_{n}\right)}{f^{\prime}\left(x_{n}\right)}, \\
x_{n+1} & =x_{n}-\frac{f\left(x_{n}\right)}{2 f^{\prime}\left(x_{n}\right)}\left[\frac{3 f^{\prime}\left(y_{n}\right)+f^{\prime}\left(x_{n}\right)}{3 f^{\prime}\left(y_{n}\right)-f^{\prime}\left(x_{n}\right)}\right] .
\end{aligned}\right.
$$

This is a well-known Jarratt's method [4].

Case 2. Now, we consider

$$
Q(x)=A x^{2}+B x+C .
$$

Then

$$
Q^{\prime}(x)=2 A x+B, \quad Q^{\prime \prime}(x)=2 A .
$$

According to Theorem 2.1, we should solve the following equations:

$$
\left\{\begin{array}{l}
A \mu^{2}+B \mu+C=1 \\
2 A \mu+B=-\frac{3}{8} \\
2 A=\frac{9}{16}
\end{array}\right.
$$

After some simplification, we get the values of $A, B, C$

$$
\left\{\begin{array}{l}
A=\frac{9}{32} \\
B=-\frac{9}{8} \\
C=2
\end{array}\right.
$$

and thus we obtain the following family of iterative methods of order four:

$$
\left\{\begin{aligned}
y_{n}= & x_{n}-\frac{2}{3} \frac{f\left(x_{n}\right)}{f^{\prime}\left(x_{n}\right)-p f\left(x_{n}\right)}, \\
x_{n+1}= & x_{n}-\frac{f\left(x_{n}\right)}{f^{\prime}\left(x_{n}\right)-p f\left(x_{n}\right)}\left[\frac{9}{32}\left(\frac{6 f^{\prime}\left(y_{n}\right)-2 f^{\prime}\left(x_{n}\right)}{3 f^{\prime}\left(x_{n}\right)-6 p f\left(x_{n}\right)}\right)^{2}\right. \\
& \left.-\frac{9}{8}\left(\frac{6 f^{\prime}\left(y_{n}\right)-2 f^{\prime}\left(x_{n}\right)}{3 f^{\prime}\left(x_{n}\right)-6 p f\left(x_{n}\right)}\right)+2\right] .
\end{aligned}\right.
$$


This is again a new general class of fourth-order optimal methods and one can easily get many new methods by choosing different values of the disposable parameter $p$.

Case 3. Since $p$ is a free disposable parameter in scheme (3.1), we can also directly derive following family.

(i) For $p=0$, we get

$$
\left\{\begin{aligned}
y_{n} & =x_{n}-\frac{2}{3} \frac{f\left(x_{n}\right)}{f^{\prime}\left(x_{n}\right)}, \\
x_{n+1} & =x_{n}-\frac{f\left(x_{n}\right)}{f^{\prime}\left(x_{n}\right)} Q\left(\frac{2 f^{\prime}\left(y_{n}\right)}{f^{\prime}\left(x_{n}\right)}-\frac{2}{3}\right) .
\end{aligned}\right.
$$

This is a special case of Zhou et al. family [1] for $m=1$.

Remark 1. The first most striking feature of this contribution is that we have developed an optimal general class of Jarratt's method for the first time which will converge even though the guess is far from root or the derivative is small in the vicinity of the required root.

Remark 2. The second most striking feature of this contribution is that our proposed family (3.3) have the same scaling factor of functions as that of Jarratt's method and does not fail even $f^{\prime}(x)=0$. Therefore, these techniques can be used as an alternative to Jarratt's technique or in the cases where Jarratt's technique is not successful.

Remark 3. Here, we should note that one can easily develop several new optimal families of Jarratt's method from scheme (3.1) by choosing different type of weight functions, permitting $f^{\prime}(x)=0$ in the vicinity of the required root.

Remark 4. Jarratt's method and Zhou et al. family of methods for simple roots are obtained as the special cases of our proposed scheme (3.1).

Remark 5 One should note that all the proposed families require one evaluations of the function and two of it's first-order derivative viz $f\left(x_{n}\right), f^{\prime}\left(x_{n}\right)$ and $f^{\prime}\left(y_{n}\right)$ per iteration. Theorem 2.1 shows that the proposed schemes are optimal with fourth-order convergence, as expected by Kung-Traub conjecture [5]. Therefore, the proposed class of methods has an efficiency index which equals 1.587 .

Remark 6. Further, it can be seen that our proposed scheme (3.1) gives very good approximation to the root when $|p|$ is small. This is because that, for small values of $p$, slope or angle of inclination of straight line with $x$-axis 
becomes smaller, i.e. as $p \rightarrow 0$, the straight line tends to $x$-axis. Therefore, by taking smaller values of $p$, we find close rival to Jarratt's method.

Remark 7. The results in Table 2 overwhelmingly support to the above remark 6 .

\section{Numerical Experiments}

In this section, we shall check the effectiveness of the new optimal methods. We employ the present method for $|p|=\frac{1}{2}$ in (3.3) denoted by MJM respectively to solve nonlinear equations given in Table 1. We compare them with existing classical Newton's method (NM), modified Newton's method for $|p|=\frac{1}{2}$ are denoted by (MNM), King's method [8] (KM), Ostrowski's method [9, 10] (OM) and Jarratt's method[4] (JM) respectively. We have shown the comparison of all methods mentioned above in the Table 2. Computations have been performed using $C++$ in double precision arithmetic. We use $\varepsilon=10^{-15}$ as tolerable error. The following stopping criteria are used for computer programs:

(i) $\left|x_{n+1}-x_{n}\right|<\epsilon$,

(ii) $\left|f\left(x_{n+1}\right)\right|<\epsilon$.

Example 1. $\sin x=0$. This equation has an infinite number of roots. It can be seen that Newton's method and Jarratt's method do not necessarily converge to the root that is nearest to the starting value. For example, Newton's method, Jarratt's method and Ostrowski's method with initial guess $x_{0}=-1.32$ converge to 3.141592741012573 respectively, while King's method at same guess converges to 6.283185482025146 far away from the required root zero. Similarly, Newton's method, Jarratt's method, King's method and Ostrowski's method with initial guess $x_{0}=1.51$ converge to -15.707962989807129 $,-6.283185482025146,-12.56637096405029$ and -9.424778223037719 respectively and so on. Our method does not exhibit this type of behaviour.

Example 2. $x e^{-x}=0$. Care must be taken when applying either method for approximating the root at $x=0$. The derivative of the function $x e^{-x}$ is zero at $x=1$, and negative for $x>1$. For any initial guess $x_{0}<0$, Newton's method, Jarratt's method, Ostrowski's method and King's method converges to the root very efficiently. For any initial guess $x>1$, the Newton iterates move away from zero. For example, Newton's method, Jarratt's method, King's method, Ostrowski's method with initial guess $x_{0}=1.1$ and 1.2 will diverge. On the other hand, our method give the required root after finite number of iterations. 


\begin{tabular}{llll|}
\hline$f(x)$ & roots & {$[\mathrm{a}, \mathrm{b}]$} \\
\hline$f_{1}(x)=\sin x$ & 0 & {$[-0.2,1.52]$} \\
$f_{2}(x)=x e^{-x}$ & 0 & {$[0,1.2]$} \\
$f_{3}(x)=e^{-x}+\sin x$ & 3.18306303024920 & {$[0,4]$} \\
$f_{4}(x)=x^{3}-4 x^{2}-10$ & 4.494939804077148 & {$[0,5]$} \\
$f_{5}(x)=x^{10}-1$ & 1 & {$[0,2]$} \\
$f_{6}(x)=e^{x^{2}+7 x-30}-1$ & 3 & {$[-3.5,3.25]$} \\
$f_{7}(x)=\tan ^{-1} x$ & 0 & {$[-1.5 .2]$} \\
$f_{8}(x)=x^{3}-\cos x+2$ & -1.172577977180481 & {$[-2,0.2]$} \\
$f_{9}(x)=x^{4}-x^{3}+11 x-7$ & 0.645023941993713 & {$[0,1]$} \\
$f_{10}(x)=\sin ^{2} x+x$ & 0 & {$[-0.1,0.5]$} \\
\hline
\end{tabular}

Table 1: Test functions

Example 3. $e^{-x}+\sin x$. This equation has an infinite number of roots. It can be seen that Newton's method does not necessarily converge to the root that is nearest to the starting value. For example, Newton's method, King's method and Ostrowski's method with initial guess $x_{0}=1.5$ converge to 9.424778223037719 while Jarratt's method converges to 6.283185482025146 far away from the required root 3.18306303024920. Similarly, Newton's method with initial guess $x_{0}=1.6$ converges to 6.283185482025146 , Ostrowski's method converges to 18.849555969238281 , King's method diverges to the root and Jarratt's method converges to the required root after three number of iterations and so on. Our methods do not exhibit this type of behaviour.

\section{Acknowledgments}

Ramandeep Behl acknowledges the financial support of CSIR, New Delhi, India. 


\begin{tabular}{|c|c|c|c|c|c|c|c|}
\hline$f(x)$ & $\begin{array}{l}\text { Initial } \\
\text { guess }\end{array}$ & $\overline{\mathrm{NM}}$ & $\begin{array}{l}\text { MNM } \\
|p|=\frac{1}{2}\end{array}$ & $\begin{array}{l}\overline{\mathrm{KM}} \\
\beta=1,\end{array}$ & $\mathrm{OM}$ & $\mathrm{JM}$ & $\begin{array}{l}\text { MJM } \\
|p|=\frac{1}{2}\end{array}$ \\
\hline \multirow[t]{5}{*}{1.} & -0.2 & $(3,6)$ & $(4,8)$ & $(3,9)$ & $(3,9)$ & $(2,6)$ & $(3,9)$ \\
\hline & -1.32 & CUR & $(4,8)$ & CUR & CUR & CUR & $(3,9)$ \\
\hline & 0.6 & $(3,6)$ & $(5,10)$ & $(3,9)$ & $(3,9)$ & $(3,9)$ & $(5,15)$ \\
\hline & 1.51 & CUR & $(5,10)$ & CUR & CUR & CUR & $(5,15)$ \\
\hline & 1.52 & CUR & $(6,12)$ & D & CUR & CUR & $(5,15)$ \\
\hline \multirow[t]{4}{*}{2.} & 0.9 & $(17,34)$ & $(8,16)$ & $\mathrm{D}$ & $(7,21)$ & $(7,21)$ & $(5,15)$ \\
\hline & 1 & $\mathrm{~F}$ & $(7,14)$ & $\mathrm{F}$ & $\mathrm{F}$ & $\mathrm{F}$ & $(7,21)$ \\
\hline & 1.1 & D & $(7,14)$ & D & D & D & $(7,21)$ \\
\hline & 1.2 & D & $(7,14)$ & D & $\mathrm{D}$ & D & $(7,21)$ \\
\hline \multirow[t]{4}{*}{3.} & 0 & $\mathrm{~F}$ & $(5,10)$ & $\mathrm{F}$ & $\mathrm{F}$ & $\mathrm{F}$ & $(4,12)$ \\
\hline & 1.5 & CUR & $(4,8)$ & CUR & CUR & CUR & $(3,9)$ \\
\hline & 1.6 & CUR & $(4,12)$ & D & CUR & CUR & $(3,9)$ \\
\hline & 2.5 & $(3,6)$ & $(4,12)$ & $(2,6)$ & $(2,6)$ & $(2,6)$ & $(3,9)$ \\
\hline \multirow[t]{4}{*}{4.} & 0 & & $(6,12)$ & & & & $(6,18)$ \\
\hline & 0.1 & $(22,44)$ & $(5,10)$ & $(13,39)$ & $(12,36)$ & $(8,24)$ & $(4,12)$ \\
\hline & 2.66 & $(34,68)$ & $(4,8)$ & & $(14,42)$ & $(11,33)$ & $(2,6)$ \\
\hline & 4.1 & $(4,8)$ & $(3,9)$ & $(2,6)$ & $(3,9)$ & $(3,9)$ & $(3,9)$ \\
\hline \multirow[t]{4}{*}{5.} & 0 & $\mathrm{~F}$ & $(12,24)$ & $\mathrm{F}$ & $\mathrm{F}$ & $\mathrm{F}$ & $(6,18)$ \\
\hline & 0.1 & $\mathrm{D}$ & $(12,24)$ & D & D & D & $(6,18)$ \\
\hline & 0.5 & $(42,84)$ & $(14,28)$ & D & $(17,51)$ & $(17,51)$ & $(9,27)$ \\
\hline & 1.5 & $(8,16)$ & $(8,16)$ & $(4,12)$ & $(4,12)$ & $(4,12)$ & $(6,18)$ \\
\hline \multirow[t]{4}{*}{6.} & -3.5 & $\mathrm{~F}$ & $(29,58)$ & $\mathrm{F}$ & $\mathrm{F}$ & $\mathrm{F}$ & $(17,51)$ \\
\hline & -1 & & $(2,4)$ & $\mathrm{D}$ & $\mathrm{D}$ & D & $(1,3)$ \\
\hline & 2.75 & $(29,58)$ & $(15,30)$ & $(7,21)$ & $(7,21)$ & $(7,21)$ & $(8,24)$ \\
\hline & 3.25 & $(7,14)$ & $(7,14)$ & $(4,12)$ & $(3,9)$ & $(4,12)$ & $(5,15)$ \\
\hline \multirow[t]{4}{*}{7.} & -0.5 & $(4,8)$ & $(5,10)$ & $(3,9)$ & $(3,9)$ & $(3,9)$ & $(3,9)$ \\
\hline & -1.5 & & $(5,10)$ & $(2,6)$ & $(2,6)$ & $(4,12)$ & $(4,12)$ \\
\hline & 0.5 & $(4,8)$ & $(5,10)$ & $(3,9)$ & $(3,9)$ & $(3,9)$ & $(4,12)$ \\
\hline & 3 & & $(6,12)$ & $(19,57)$ & $(5,15)$ & $\mathrm{D}$ & $(5,15)$ \\
\hline \multirow[t]{3}{*}{8.} & -2 & $(5,10)$ & $(6,12)$ & $(3,9)$ & $(3,9)^{\prime}$ & $(3,9)$ & $(3,9)^{\prime}$ \\
\hline & -1 & $(4,12)$ & $(4,12)$ & $(2,6)$ & $(2,6)$ & $(3,9)$ & $(3,9)$ \\
\hline & 0 & & $(15,30)$ & & & & $(8,24)$ \\
\hline \multirow[t]{2}{*}{9.} & 0 & $(3,9)$ & $(4,12)$ & $(2,6)$ & $(2,6)$ & $(2,6)$ & $(2,6)$ \\
\hline & 1 & $(3,9)$ & $(4,12)$ & $(3,9)$ & $(3,9)$ & $(3,9)$ & $(2,6)$ \\
\hline \multirow[t]{2}{*}{10} & -0.1 & $(4,12)$ & $(4,12)$ & $(3,9)$ & $(3,9)$ & $(3,9)$ & $(3,9)$ \\
\hline & 0.5 & $(5,15)$ & $(6,18)$ & $(4,12)$ & $(4,12)$ & $(4,12)$ & $(4,12)$ \\
\hline
\end{tabular}

Table 2: (Total number of iterations to approximate the zero of a function, total number of function evaluations for various multipoint iterative methods)

CUR: converge to undesired root, D: divergent, F: method fails.

Note. The numerical examples considered above show that in many cases our method is an efficeint alternative to Jarratt's method, which may converge to undesired root or even fail. 


\section{References}

[1] X. Zhou, X. Chen, Y. Song, Constructing higher-order methods for obtaining the muliplte roots of nonlinear equations, J. Comput. Appl. Math., 235 (2011), 4199-4206.

[2] S. Kumar, V. Kanwar, S. K. Tomar, S. Singh, Geometrically constructed families of Newton's method for unconstrained optimization and nonlinear equations, Int. J. Math. Math. Sci. (2011), doi: 10.1155/2011/972537.

[3] Mamta V. Kanwar, V.K. Kukreja, S. Singh, On a class of quadratically convergent iteration formulae, Appl. Math. Comput., 166 (2005), 633-637.

[4] P. Jarratt, Some efficient fourth-order multipoint methods for solving equations, BIT (1969), 119-124.

[5] H.T. Kung, J.F. Taub, Optimal order of one-point and multipoint iteration, Journal of the ACM, 21 (1974), 643-651.

[6] V. Kanwar, S. K. Tomar, Modified families of Newton, Halley and Chebyshev methods, Appl. Math. Comput., 192 (2007), 20-26.

[7] X. Wu, A new continuation Newton-like method and its deformation, Appl. Math. Comput., 112 (2000), 75-78.

[8] R.F. King, A family of fourth order methods for nonlinear equations, SIAM J. Numer. Anal., 10 (1973), 876-879.

[9] V. Kanwar, R. Behl, Kapil K. Sharma, Simply constructed family of a Ostrowski's method with optimal order of convergence, Comput. Math. Appl., 62 (2011), 4021-4027.

[10] A.M. Ostrowski, Solutions of Equations and System of Equations, Academic Press, New York (1960).

[11] J.F. Traub, Iterative Methods for the Solution of Equations, Prentice-Hall, Englewood Cliffs, NJ (1964). 
